\title{
DISPONIBILIDADE DE CÁDMIO E CHUMBO PARA MILHO EM SOLO ADUBADO COM FERTILIZANTES FOSFATADOS ${ }^{(1)}$
}

\author{
Eriberto Vagner de Souza Freitas ${ }^{(2)}$, Clístenes Williams Araújo do \\ Nascimento $^{(3)}$, Daniel Franco Goulart ${ }^{(4)}$ \& João Paulo Siqueira da Silva ${ }^{(4)}$
}

\begin{abstract}
RESUMO
Fertilizantes fosfatados são utilizados intensamente na agricultura, pois a baixa disponibilidade de $\mathbf{P}$ frequentemente limita o rendimento das culturas nas condições brasileiras. Esses fertilizantes, entretanto, constituem uma via de entrada de metais pesados no solo. Este trabalho objetivou avaliar o potencial de contaminação do solo por $\mathrm{Cd}$ e $\mathrm{Pb}$ adicionados por diferentes fertilizantes fosfatados, bem como a absorção destes por plantas de milho. Foram aplicadas cinco doses de diferentes fontes de P: superfosfato simples, superfosfato triplo, fosfato de Araxá, termofosfato de Yoorin e fosfato natural de Gafsa. As doses de $\mathrm{P}$ equivaleram a 0, 100, 300, 500 e $800 \mathrm{~kg} \mathrm{ha}^{-1} \mathrm{de}_{2} \mathrm{O}_{5}$. Dois cultivos sucessivos com milho foram conduzidos no solo. $\mathrm{O}$ fosfato natural de Gafsa apresentou os maiores teores de $\mathrm{Cd}$ e $\mathrm{Pb}$. Entre os fertilizantes acidulados, o superfosfato simples apresentou maior teor de $\mathrm{Cd} \mathrm{e} \mathrm{Pb}$, e o termofosfato, maior concentração de $\mathrm{Pb}$ do que os acidulados. A aplicação de fosfato de Gafsa proporcionou as maiores concentrações de $\mathbf{P b}$ na parte aérea do milho no primeiro cultivo. Este fosfato também foi responsável pelo maior teor de $\mathrm{Cd}$ nas plantas no segundo cultivo. $\mathrm{O}$ ácido cítrico foi mais eficiente em prever os teores disponíveis de $\mathrm{Cd}$, enquanto o DTPA estimou melhor os teores de Pb.
\end{abstract}

Termos de indexação: metais pesados, fertilizantes minerais, contaminação de solo.

\footnotetext{
(1) Recebido para publicação em julho de 2008 e aprovado em agosto de 2009.

${ }^{(2)}$ Doutorando do Programa de Pós-Graduação em Ciência do Solo do Departamento de Agronomia - Universidade Federal Rural de Pernambuco - UfRPE. R. Dom Manoel de Medeiros s/n, Dois Irmãos, CEP 52171-900 Recife (PE). Bolsista do CNPq. Email: eribertovagner@yahoo.com.br

(3) Professor do Departamento de Agronomia, UFRPE. Pesquisador do CNPq. E-mail: clistenes@pq.cnpq.br

(4) Graduando em Agronomia, UFRPE. E-mail: jpsp@yahoo.com.br; danielgoulart_pr@hotmail.com
} 


\title{
SUMMARY: CADMIUM AND LEAD AVAILABILITY TO CORN IN SOIL AMENDED WITH PHOSPHORUS FERTILIZERS
}

\begin{abstract}
Phosphorus fertilizers are intensively used in Brazil, since the low availability of phosphorus often limits yields in tropical soils. However, these fertilizers can be a entranceway for soil contamination with heavy metals. This study was carried out to investigate heavy metal contamination caused by the application of five different phosphorus fertilizers as well as the metal uptake by corn (Zea mays) plants. The fertilizers simple superphosphate, triple superphosphate, Araxá rock phosphate, Yoorin thermophosphate, and Gafsa rock phosphate were applied at rates of 0,100, 300, 500 and $800 \mathrm{~kg} \mathrm{ha}^{-1} \mathrm{P}_{2} \mathrm{O}_{5}$ in two successive corn cycles. The highest $\mathrm{Cd}$ and $\mathrm{Pb}$ concentrations were found in Gafsa rock phosphate. For the acidulated phosphates, simple superphosphate was the richest in $\mathrm{Cd}$ and $\mathrm{Pb}$, while the $\mathrm{Pb}$ concentration was highest in Yoorin thermophosphate. Gafsa application induced the highest Pb concentration in corn shoots in the first crop cycle. This fertilizer was also responsible for the highest Cd concentration in plants in the second crop. Citric acid was the most efficient extractant for predicting the available soil Cd contents, whereas DTPA estimated Pb availability best.
\end{abstract}

Index terms: heavy metals, inorganic fertilizers, soil contamination.

\section{INTRODUÇÃO}

A contaminação de solos por metais pesados compromete a sustentabilidade agrícola e ambiental, podendo apresentar sérias consequências à saúde humana em decorrência da contaminação da cadeia trófica via absorção vegetal. Em uma lista de 20 substâncias tóxicas consideradas mais perigosas pela Agência de Proteção Ambiental dos EUA, o Pb e o Cd ocupam lugar de destaque, sendo o segundo e o quarto, respectivamente, entre os metais pesados (ATSDR, 1997). Contaminação de solos por esses elementos pode causar diversos problemas, incluindo contaminação de lençol freático e toxidez em plantas e animais.

Em solos agrícolas, as principais vias de contaminação por metais pesados são representadas por resíduos industriais, aplicação de lodo e fertilizantes fosfatados (McLaughlin \& Singh, 1999). Fertilizantes nitrogenados e potássicos geralmente contêm concentrações de metais pesados muito menores que as encontradas nos fosfatados (McBride \& Spiers, 2001) e representam pequena possibilidade de contaminação. Fosfatos de rocha constituem a matéria-prima para a fabricação dos fertilizantes fosfatados acidulados e são amplamente utilizados in natura como fonte de P. É conhecido que esses fosfatos contêm naturalmente teores de vários metais pesados, que podem ser de preocupação quanto à contaminação ambiental (Agbenin, 2002) e que, no processo de produção de fontes mais solúveis de $\mathrm{P}$, a maior parte dos metais permanece no fertilizante. Por exemplo, Mortvedt (1987) observou que $60 \%$ do Cd presente em superfosfato triplo foi remanescente da própria rocha fosfática. Em trabalho recente, Bizarro et al. (2008) encontraram teor de $\mathrm{Cd}$ em fontes aciduladas de $\mathrm{P}$, superfosfatos simples e triplo, variando de 2 a 13 e 3 a $33 \mathrm{mg} \mathrm{kg}^{-1}$, respectivamente.
Devido aos teores relativamente baixos dos metais $\mathrm{Cd}$ e $\mathrm{Pb}$ em fertilizantes comerciais, muitos pesquisadores têm recomendado sua utilização sem restrições quanto a problemas de contaminação ambiental. No entanto, pouca informação está disponível sobre a absorção desses metais por plantas em solos adubados com diferentes fertilizantes fosfatados e o efeito de acumulação desses elementos no solo a longo prazo. Adições globais de metais pesados ao solo por fertilizantes são da ordem de $30.000-250.000 \mathrm{~kg} \mathrm{ano}^{-1} \mathrm{de}$ $\mathrm{Cd}$ e $420.000-2.300 .000 \mathrm{~kg} \mathrm{ano}^{-1}$ de $\mathrm{Pb}$ (Nriagu \& Pacyna, 1988). A legislação internacional apresenta ampla faixa de variações em relação aos valores de referência de metais em fertilizantes: nos Estados Unidos os teores máximos permitidos de $\mathrm{Cd}$ e $\mathrm{Pb}$ em fertilizantes fosfatados são de 10 e $61 \mathrm{mg} \mathrm{kg}^{-1}$, respectivamente (Westfall et al., 2005); no Japão e na China, de 8 e $100 \mathrm{mg} \mathrm{kg}^{-1}$ de $\mathrm{Cd}$ e $\mathrm{Pb}$, respectivamente; na Austrália o teor de Cd permitido nos fertilizantes fosfatados chega a $300 \mathrm{mg} \mathrm{kg}^{-1}$ (AFPC, 2006). No Brasil, a Instrução Normativa número 27 de 2006, do Ministério da Agricultura, Pecuária e Abastecimento, estabelece limites máximos de metais pesados de $4 \mathrm{mg} \mathrm{kg}^{-1}$ de $\mathrm{Cd}$ e $20 \mathrm{mg} \mathrm{kg}^{-1}$ de $\mathrm{Pb}$ por ponto percentual de $\mathrm{P}_{2} \mathrm{O}_{5}$ em fertilizantes fosfatados (Brasil, 2006).

A determinação das concentrações de $\mathrm{Cd}$ e $\mathrm{Pb}$ nos fertilizantes e métodos adequados de extração desses metais no solo é importante para monitorar riscos de contaminação e poluição desses metais no ambiente. Diversos métodos de extração são empregados na quantificação dos teores disponíveis de metais no solo, porém, muitas vezes, os valores encontrados variam amplamente entre os extratores. A variação da quantidade extraída deve-se às diferentes maneiras de atuação dos diferentes extratores e às características do solo que alteram a disponibilidade dos metais pesados (Nascimento et al., 2002). 
Os fertilizantes fosfatados são utilizados intensamente na agricultura, pois a baixa disponibilidade de $\mathrm{P}$ frequentemente limita $\mathrm{O}$ rendimento das culturas nas condições brasileiras. Esses fertilizantes constituem uma via de entrada de metais pesados na cadeia alimentar. Este trabalho objetivou avaliar o potencial de contaminação de Cd e $\mathrm{Pb}$ adicionados ao solo por diferentes fertilizantes fosfatados e a estimativa de sua biodisponibilidade por extratores químicos, bem como a absorção dos metais por plantas de milho (Zea mays L.).

\section{MATERIAL E MÉTODOS}

O experimento, realizado em casa de vegetação, utilizou um solo classificado como Argissolo VermelhoAmarelo textura média, coletado da camada de 0$20 \mathrm{~cm}$ e caracterizado química e fisicamente (Embrapa, 1997, 1999) (Quadro 1). Os teores totais de $\mathrm{Cd}$ e $\mathrm{Pb}$ nos fertilizados fosfatados foram obtidos por digestão nítrico-perclórica $(3: 1 \mathrm{v} / \mathrm{v})$ de amostras maceradas (Quadro 2).

O solo seco ao ar, destorroado e homogeneizado foi passado em peneira de $4 \mathrm{~mm}$ de abertura de malha. A acidez foi corrigida para $\mathrm{pH}$ na faixa de $6,0-6,2$, utilizando-se carbonato de cálcio e óxido de Mg P.A. (na proporção molar de 3:1), em quantidades previamente definidas em ensaio de incubação. $\mathrm{O}$ solo foi adubado, aplicando-se em cada vaso doses equivalentes a $0,100,300,500$ e $800 \mathrm{~kg} \mathrm{ha}^{-1}$ de $\mathrm{P}_{2} \mathrm{O}_{5}$ de cinco diferentes fontes de P: superfosfato simples (SS), superfosfato triplo (ST), termofosfato Yoorin (TY), fosfato de Araxá (FA) e fosfato natural de Gafsa (FG). Duas plantas de milho, cultivar AG1051, foram cultivadas em cada vaso plástico com $3 \mathrm{dm}^{3}$ de solo.

$\mathrm{O}$ experimento foi conduzido por um período de 30 dias após o desbaste, o qual foi efetuado sete dias após a semeadura, mantendo-se duas plantas por vaso. Uma adubação adicional foi realizada 10 dias após a germinação, aplicando-se em cada vaso as seguintes doses de nutrientes: $200 \mathrm{mg} \mathrm{dm}^{-3}$ de $\mathrm{N}\left(\left(\mathrm{NH}_{4}\right)_{2} \mathrm{SO}_{4} \mathrm{e}\right.$
Quadro 1. Principais características químicas e físicas do solo utilizado no experimento

\begin{tabular}{|c|c|}
\hline Característica & Valor \\
\hline pH (água 1:2,5) & 5,30 \\
\hline $\mathrm{Al}^{3+}\left(\mathrm{cmol}_{\mathrm{c}} \mathrm{dm}^{-3}\right)$ & 0,82 \\
\hline $\mathrm{Ca}^{2+}\left(\mathrm{cmol}_{\mathrm{c}} \mathrm{dm}^{-3}\right)$ & 1,17 \\
\hline $\mathrm{Mg}^{2+}\left(\mathrm{cmol}_{\mathrm{c}} \mathrm{dm}^{-3}\right)$ & 0,73 \\
\hline $\mathrm{P}\left(\mathrm{mg} \mathrm{dm} \mathrm{m}^{-3}\right)$ & 2,82 \\
\hline $\mathrm{K}\left(\mathrm{cmol}_{\mathrm{c}} \mathrm{dm}^{-3}\right)$ & 0,11 \\
\hline $\mathrm{Na}\left(\mathrm{cmol}_{\mathrm{c}} \mathrm{dm}^{-3}\right)$ & 0,23 \\
\hline $\mathrm{H}+\mathrm{Al}\left(\mathrm{cmol}_{\mathrm{c}} \mathrm{dm}^{-3}\right)$ & 3,99 \\
\hline $\mathrm{CO}\left(\mathrm{g} \mathrm{kg}^{-1}\right)$ & 9,80 \\
\hline $\mathrm{Cd}\left(\mathrm{mg} \mathrm{dm}^{-3}\right)$ & nd \\
\hline $\mathrm{Pb}\left(\mathrm{mg} \mathrm{dm}^{-3}\right)$ & 0,01 \\
\hline Areia $\left(\mathrm{g} \mathrm{kg}^{-1}\right)$ & 707 \\
\hline Silte $\left(\mathrm{g} \mathrm{kg}^{-1}\right)$ & 44 \\
\hline Argila (g kg-1) & 249 \\
\hline $\mathrm{Ds}\left(\mathrm{g} \mathrm{cm}^{-3}\right)$ & 1,09 \\
\hline Capacidade de campo ( $\left.\mathrm{g} \mathrm{g}^{-1}\right)$ & 0,18 \\
\hline
\end{tabular}

CO: carbono orgânico; Ds: densidade do solo; nd: não detectado.

$\left.\mathrm{KNO}_{3}\right)$ e $150 \mathrm{mg} \mathrm{dm}{ }^{-3} \mathrm{de} \mathrm{K}\left(\mathrm{KNO}_{3}\right)$. Os vasos foram irrigados com água destilada diariamente, mantendo os solos a aproximadamente $80 \%$ da capacidade de retenção de água, por meio de pesagens e reposição da água perdida por evapotranspiração.

Com o objetivo de avaliar o efeito do tempo de contato solo-fertilizante e a capacidade de absorção residual dos metais $\mathrm{Cd}$ e $\mathrm{Pb}$, um segundo cultivo com milho foi conduzido no solo, com duração de 30 dias. Ao final de cada cultivo, foi realizada a coleta das raízes e da parte aérea das plantas. No entanto, apenas as massas da matéria seca da parte aérea foram obtidas, as quais, após secagem em estufa a $65^{\circ} \mathrm{C}$, foram trituradas e submetidas à digestão nítrico-perclórica (Embrapa, 1999) para quantificação de Cd e Pb por espectrofotômetro de absorção atômica.

Quadro 2. Valores médios de cádmio e chumbo nos fertilizantes fosfatados e quantidade dos metais pesados incorporados por hectare de solo pela aplicação de fertilizantes fosfatados

\begin{tabular}{|c|c|c|c|c|}
\hline \multirow[t]{2}{*}{ Fertilizante } & \multicolumn{2}{|c|}{ Cádmio } & \multicolumn{2}{|c|}{ Chumbo } \\
\hline & $\mathrm{mg} \mathrm{kg}^{-1(1)}$ & $\mathrm{g} \mathrm{ha}^{-1(2)}$ & $\mathrm{mg} \mathrm{kg}^{-1(1)}$ & $\mathrm{g} \mathrm{ha}^{-1(2)}$ \\
\hline Superfosfato Simples & $15 \pm(2)$ & 1,50 & $54 \pm(4)$ & 5,40 \\
\hline Superfosfato Triplo & $14 \pm(4)$ & 1,40 & $21 \pm(3)$ & 2,10 \\
\hline Termofosfato Yoorin & $10 \pm(1)$ & 1,00 & $99 \pm(1)$ & 9,90 \\
\hline Fosfato de Araxá & $8 \pm(1)$ & 0,80 & $49 \pm(4)$ & 4,90 \\
\hline Fosfato de Gafsa & $35 \pm(2)$ & 3,50 & $234 \pm(10)$ & 23,40 \\
\hline
\end{tabular}

(1) Teor médio dos metais nos fertilizantes. ${ }^{(2)}$ Valores considerando uma aplicação a lanço de uma dose de 100 kg ha ${ }^{-1}$ de $\mathrm{P}_{2} \mathrm{O}_{5}$. Valores entre parênteses correspondem ao desvio-padrão das médias. 
A coleta de amostras do solo homogeneizado foi realizada ao final de cada cultivo. Subamostras de TFSA foram retiradas para determinação do teor disponível dos metais $\mathrm{Cd}$ e $\mathrm{Pb}$ pelos extratores químicos DTPA (Lindsay \& Norvell, 1978) e ácido cítrico $10 \mathrm{mmol} \mathrm{L}{ }^{-1}$, com uma relação solo:solução de 1:10 e agitação por cinco minutos, proposta pelo presente trabalho. Os teores de $\mathrm{Cd}$ e $\mathrm{Pb}$ foram determinados por espectrofotômetro de absorção atômica.

Os tratamentos foram arranjados em esquema fatorial $5 \times 5$, correspondendo a cinco fontes de fertilizantes fosfatados e cinco doses destes, dispostos em delineamento experimental de blocos casualizados, com três repetições. Os dados foram submetidos à análise de variância e as equações de regressão ajustadas em função das doses de fertilizantes aplicadas no solo e do teor dos metais na parte aérea das plantas, sendo a escolha do modelo feita com base na significância dos estimadores dos parâmetros e nos maiores valores de $\mathrm{R}^{2}$. A eficiência dos extratores químicos em prever a biodisponibilidade dos metais pesados foi avaliada por meio da correlação de Pearson entre os teores obtidos em solo pelos extratores ácido cítrico e DTPA e aqueles determinados na parte aérea do milho. Todas as análises estatísticas foram realizadas no Sistema para Análises Estatísticas SAEG 9.1 da UFV.

\section{RESULTADOS E DISCUSSÃO}

\section{Teor de cádmio e chumbo nos fertilizantes fosfatados}

Os fosfatos naturais apresentaram características marcadamente diferentes quanto à concentração dos metais pesados (Quadro 2). O teor de $\mathrm{Pb}$ no fosfato de Araxá encontra-se na faixa de valores citados na literatura (Amaral Sobrinho et al., 1992; Mendes et al., 2006), bem abaixo dos teores encontrados no fosfato natural de Gafsa, com valor médio de $234 \mathrm{mg} \mathrm{kg}^{-1}$. Com relação ao $\mathrm{Cd}$, o teor médio encontrado foi abaixo dos valores determinados pelos trabalhos citados anteriormente. Os resultados levam a concordar com a afirmativa (Langenbach \& Sarpa, 1985; Camargo et al., 2000) de que os fosfatos naturais brasileiros apresentam baixos teores de $\mathrm{Cd}$.

É possível observar que os teores de $\mathrm{Cd}$ e $\mathrm{Pb}$ no superfosfato triplo estão abaixo dos encontrados em outros trabalhos (Prochnow et al., 2001; Mendes et al., 2006), especialmente o teor de Cd (Bizarro et al., 2008). De acordo com Prochnow (2002), no caso dos superfosfatos, a matéria-prima básica é a rocha fosfática, que, quando atacada por ácido sulfúrico, dá origem ao superfosfato simples e, atacada por ácido fosfórico, origina o superfosfato triplo. Quando no processo de produção dos fertilizantes fosfatados totalmente acidulados utilizam-se rochas fosfáticas ou concentrados apatíticos (produto do processo de beneficiamento de rochas que contêm impurezas) de elevada qualidade, são obtidos fertilizantes formados praticamente pelos compostos de interesse da indústria. A obtenção de fertilizantes de elevada solubilidade e concentração de $\mathrm{P}$ traz, como consequência, produtos mais "puros" no tocante à contaminação de elementos metálicos. O termofosfato Yoorin apresentou teor de $\mathrm{Cd}$ maior apenas que o do fosfato natural de Araxá entre os fertilizantes, porém o teor de $\mathrm{Pb}$ só foi menor que o do fosfato de Gafsa. Os teores médios de $\mathrm{Pb}$ foram maiores que os de $\mathrm{Cd}$ em todos os fertilizantes utilizados no estudo. Os teores encontrados dos metais, tanto para o superfosfato triplo como para o simples, estão abaixo dos teores permitidos pela Instrução Normativa $\mathrm{n}^{0} 27$ do Ministério da Agricultura, Pecuária e Abastecimento, que são de $4 \mathrm{mg} \mathrm{kg}^{-1}$ de Cd e $20 \mathrm{mg} \mathrm{kg}^{-1}$ de $\mathrm{Pb}$ por ponto percentual de $\mathrm{P}_{2} \mathrm{O}_{5}$ em fertilizantes fosfatados (Brasil, 2006).

Os resultados do trabalho indicam que o uso de doses agronômicas desses fertilizantes fosfatados não representa risco de aumentar a concentração de $\mathrm{Pb}$ muito acima dos teores naturalmente encontrados em solos, corroborando outros trabalhos (Otero et al., 2005; Mendes et al., 2006; Bizarro et al., 2008). Portanto, considerando uma profundidade de incorporação de $20 \mathrm{~cm}$, seriam necessárias 1.764 aplicações da mais alta dose de fosfato de Gafsa utilizada neste experimento, que apresenta a maior concentração de $\mathrm{Pb}$, para elevar os teores no solo ao nível de intervenção do metal em solos agrícolas (180 $\mathrm{mg} \mathrm{kg}^{-1}$ ) orientado pela CETESB (2005). Considerando doses agronomicamente aplicadas de, por exemplo, $5 \mathrm{t} \mathrm{ha}^{-1}$ na fosfatagem, esse teor no solo poderia ser atingido em 282 aplicações. Evidentemente, deve-se considerar a retirada de $\mathrm{Pb}$ pela planta e a possibilidade de sua perda por lixiviação em solos mais arenosos. O fosfato de Gafsa também apresenta a maior concentração de $\mathrm{Cd}$ entre os fertilizantes testados (Quadro 2). No entanto, seriam necessárias 197 aplicações da maior dose do FG para aumentar a concentração do $\mathrm{Cd}$ no solo ao nível de intervenção proposto pela CETESB (2005), que é de $3 \mathrm{mg} \mathrm{kg}^{-1}$ do metal. Considerando novamente a aplicação de $5 \mathrm{t} \mathrm{ha}^{-1}$ na fosfatagem, este teor no solo poderia ser atingido em 32 aplicações. Embora os resultados indiquem segurança no uso de fertilizantes quanto à contaminação por $\mathrm{Cd} \mathrm{e} \mathrm{Pb}$, é importante monitorar o seu uso a fim de manter a qualidade de solo.

\section{Biodisponibilidade de cádmio e chumbo}

Considerando a maior dose ( $800 \mathrm{~kg} \mathrm{ha}^{-1} \mathrm{de}_{2} \mathrm{O}_{5}$ ) dos fertilizantes fosfatados, o termofosfato de Yoorin foi o mais eficiente, entre todos, em promover a translocação dos metais $\mathrm{Cd}$ e $\mathrm{Pb}$ para a parte aérea do milho nos dois cultivos, com valores médios de 0,07 e $0,75 \mathrm{mg} /$ vaso de $\mathrm{Cd}$ e de 0,63 e $1,61 \mathrm{mg} /$ vaso de $\mathrm{Pb}$, respectivamente no primeiro e segundo cultivos. Isso se deve ao fato de o fertilizante apresentar a segunda maior concentração de $\mathrm{Pb}$ e as diferenças entre os 
teores de Cd nos fertilizantes não serem tão marcantes (Quadro 2). O superfosfato simples foi a segunda fonte mais eficiente na elevação do conteúdo de $\mathrm{Cd}$ e $\mathrm{Pb}$ na parte aérea do milho nos dois cultivos. Considerando a maior dose, o conteúdo médio foi de 0,06 e $0,32 \mathrm{mg} /$ vaso de $\mathrm{Cd}$ e de 0,47 e $1,07 \mathrm{mg} /$ vaso de $\mathrm{Pb}$, respectivamente no primeiro e segundo cultivos. Os resultados mostram que os metais $\mathrm{Cd}$ e $\mathrm{Pb}$ podem entrar na cadeia trófica via absorção pelas plantas com a aplicação de fertilizantes; é importante monitorar o seu uso, a fim de manter a qualidade dos produtos de origem vegetal e, indiretamente, animal.

A aplicação dos fertilizantes fosfatados acidulados influenciou significativamente os teores de $\mathrm{Cd}$ e $\mathrm{Pb}$ na parte aérea das plantas de milho em ambos os cultivos (Figura 1). Os valores de $\mathrm{Pb}$ na parte aérea do milho foram maiores que os de $\mathrm{Cd}$ nas fontes aciduladas nos dois cultivos, devido à maior concentração de $\mathrm{Pb}$ nos fertilizantes (Quadro 2). Os resultados discordam
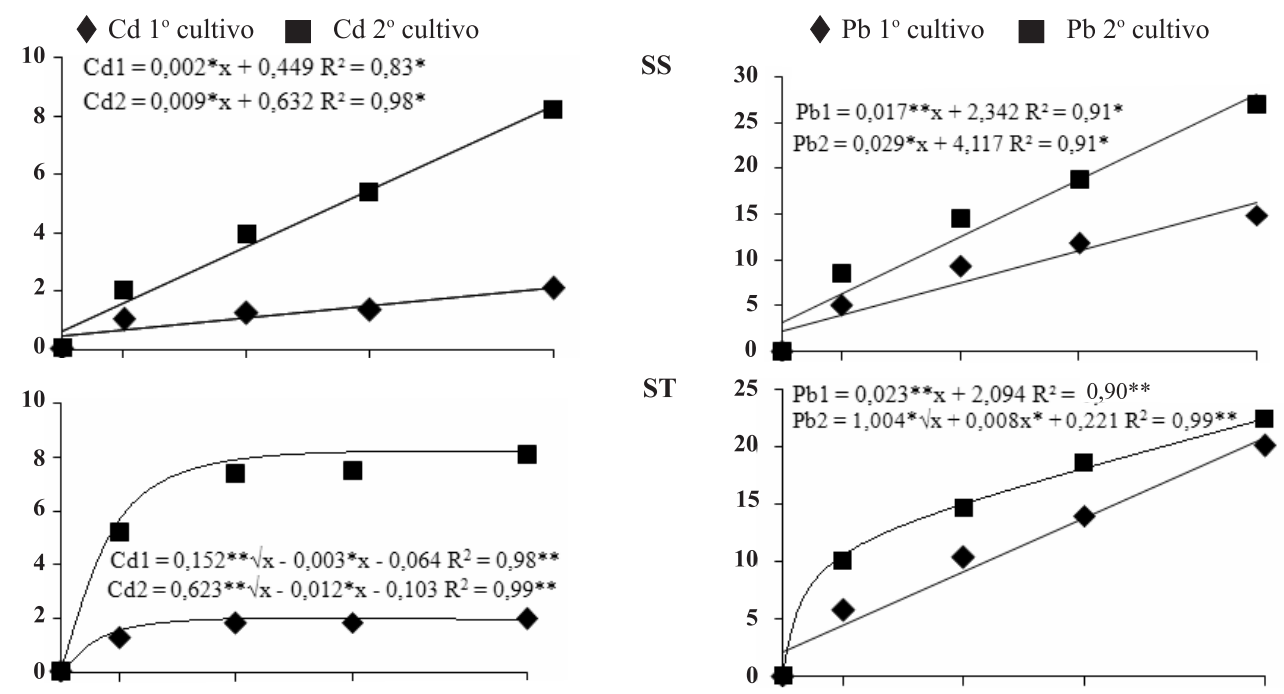

ST
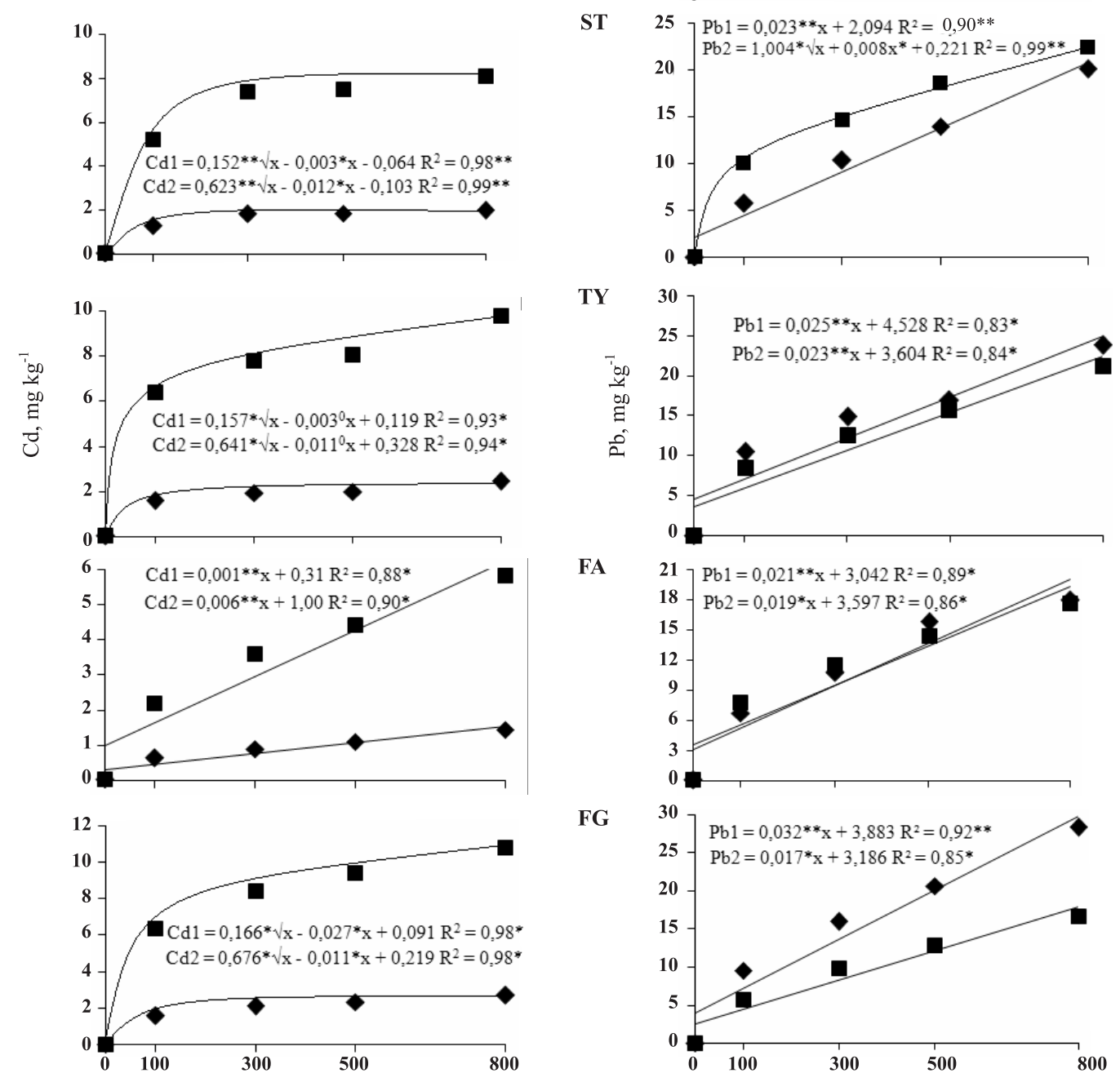

DOSE DE $\mathrm{P}_{2} \mathrm{O}_{5}, \mathrm{~kg} \mathrm{ha}^{-1}$

Figura 1. Teores de Cd e Pb na parte aérea das plantas de milho em dois cultivos sucessivos em função das doses dos fertilizantes superfosfato simples (SS), superfosfato triplo (ST), termofosfato Yoorin (TY), fosfato natural de Araxá (FA) e fosfato natural de Gafsa (FG) . **, * e $^{\circ}$ : $\operatorname{significativos~a~} 1,5$ e $10 \%$, respectivamente. 
dos encontrados por Mendes et al. (2006), que não detectaram $\mathrm{Pb}$ na parte aérea de plantas de mucunapreta em dois cultivos.

$\mathrm{O}$ aumento das doses dos fertilizantes fosfatados proporcionou maior incremento na concentração de $\mathrm{Pb}$ na parte aérea das plantas, no segundo cultivo, para todas as fontes de $\mathrm{P}$ utilizadas, exceto no termofosfato Yoorin (Figura 1). A aplicação de superfosfato simples e triplo, nas doses de 100 e $300 \mathrm{~kg} \mathrm{ha}^{-1}$ de $\mathrm{P}_{2} \mathrm{O}_{5}$, aumentou a concentração de $\mathrm{Pb}$ na matéria seca da parte aérea em 10 e 15 vezes no segundo cultivo plantado, respectivamente, quando comparado ao solo em que não foram aplicados fertilizantes.

O incremento da concentração de $\mathrm{Cd}$ na parte aérea das plantas foi significativamente maior do primeiro para o segundo cultivo, com a aplicação de todas as fontes de $\mathrm{P}$, mas não superior aos teores de $\mathrm{Pb}$. $\mathrm{Os}$ resultados do superfosfato triplo corroboram os encontrados por Mendes et al. (2006), que observaram maior concentração de $\mathrm{Cd}$ na parte aérea de mucunapreta no segundo cultivo do que no primeiro. A fertilização com superfosfato simples, triplo e termofosfato de Yoorin na maior dose $\left(800 \mathrm{~kg} \mathrm{ha}^{-1} \mathrm{de}\right.$ $\mathrm{P}_{2} \mathrm{O}_{5}$ ) aumentou a concentração de Cd na matéria seca da parte aérea de milho em 8,8 e 10 vezes, respectivamente, comparando-se aos tratamentos sem adubação. A alta solubilidade desses produtos pode promover absorção de metais por plantas, como mostram os trabalhos de Gonçalves Júnior et al. (2000) e Chien et al. (2003), os quais observaram acumulação de Cd na parte aérea de plantas de soja e milho, respectivamente, devido à aplicação de superfosfatos solúveis.

O incremento das doses dos fosfatos naturais promoveu significativo aumento na concentração de Cd na parte aérea das plantas nos dois cultivos (Figura 1). O maior tempo de contato dos fertilizantes no solo, especialmente fosfato de Araxá, que é considerado de baixa reatividade aumentou a solubilidade do $\mathrm{Cd}$ - evidenciado pelo aumento significativo do metal absorvido pelas plantas no segundo cultivo. Os resultados corroboram Mendes et al. (2006) no que se refere ao primeiro cultivo realizado para os fosfatos de Araxá e Gafsa, mas discordam dos observados no segundo cultivo, pois o Cd não foi detectado neste. O fosfato de Araxá apresentou a menor concentração de Cd na matéria seca da parte aérea das plantas entre todos os fertilizantes deste experimento, o que é explicado pelas características químicas do produto, visto que o fosfato apresenta a menor reatividade e menor concentração de Cd na sua constituição.

A aplicação do fosfato natural de Araxá em doses crescentes não promoveu diferença entre os cultivos do milho, mas aumentou a concentração média de $\mathrm{Pb}$ na parte aérea das plantas em $7,11,16$ e 18 vezes para as doses de 100, 300, 500 e $800 \mathrm{~kg} \mathrm{ha}^{-1} \mathrm{de}_{2} \mathrm{O}_{5}$, respectivamente (Figura 1). Entre todos os fertilizantes, o fosfato de Araxá disponibilizou o menor teor de $\mathrm{Pb}$ para as plantas, confirmando que os principais fosfatos naturais brasileiros apresentam baixos teores de metais pesados (Langenbach \& Sarpa, 1985; Camargo et al., 2000).

Contrariamente a todos os fertilizantes fosfatados deste trabalho, o fosfato natural de Gafsa promoveu maior aumento de $\mathrm{Pb}$ nas plantas de milho no primeiro cultivo em relação ao segundo (Figura 1). Na classificação de fosfatos naturais, o fosfato de Gafsa de origem sedimentar é considerado "mole" ou reativo, pois apresenta teor de $\mathrm{P}_{2} \mathrm{O}_{5}$ solúvel em ácido cítrico igual ou superior a $30 \%$ do teor total de $\mathrm{P}_{2} \mathrm{O}_{5}(\mathrm{Braga}$ et al., 1991).

Pode ser observado que as diferenças nas amplitudes dos teores de Cd entre os cultivos foram mais marcantes do que as do $\mathrm{Pb}$ em todos os fertilizantes. Sabe-se que as características de solubilidade das fontes de P são de grande importância em relação à sua eficiência, e os fosfatos de maior solubilidade, sendo mais prontamente disponíveis, favoreceriam a liberação e absorção dos elementos contidos em curto prazo (Bedin et al., 2003). Os fosfatos naturais "moles" ou reativos, que reagem no solo mais lentamente, podem apresentar efeito compensatório na disponibilização dos elementos por períodos mais longos, podendo ser equivalentes aos fertilizantes mais solúveis, devido à perda do poder fertilizante destes (Kaminski \& Peruzzo, 1997). No que se refere à disponibilidade dos metais no solo, o Cd tende a ser mais móvel em solos e, portanto, mais disponível às plantas, quando comparado ao $\mathrm{Pb}$, que é considerado um dos metais pesados menos móveis; isso explica as diferenças mais marcantes nas concentrações de $\mathrm{Cd}$ do primeiro cultivo para o segundo do que de $\mathrm{Pb}$.

\section{Disponibilidade dos metais pesados no solo}

Para ambos os metais, nos dois cultivos, os teores disponíveis extraídos por ácido cítrico e DTPA, foi observado aumento linear e significativo com o aumento das doses dos fertilizantes fosfatados (Quadro 3). A disponibilidade de Cd extraído por ácido cítrico no solo aumentou do primeiro para o segundo cultivo. Já os teores disponíveis de Pb-DTPA diminuíram do primeiro para o segundo cultivo. Isso explicaria os maiores incrementos de $\mathrm{Cd}$ em relação a $\mathrm{Pb}$ na parte aérea das plantas do primeiro para o segundo cultivo. Além dos metais pesados, os fertilizantes fosfatados, evidentemente, liberam P. De acordo com Cotter-Howells \& Caporn (1996), o P presente na forma de íon livre, $\mathrm{HPO}_{4}{ }^{2-}$, na solução do solo pode formar complexos fosfato metal, reduzindo dessa forma a disponibilidade de $\mathrm{Pb}$ do primeiro para o segundo cultivo. Com relação ao $\mathrm{Cd}$, os resultados da literatura são controversos. Krishnamurti et al. (1999) constataram que a fertilização fosfatada poderia aumentar a disponibilidade de Cd para as plantas. 
O ácido cítrico foi mais eficiente em avaliar a disponibilidade de $\mathrm{Cd}$ no solo para todos os fertilizantes, sendo pouco eficiente para $\mathrm{Pb}$. O DTPA foi eficiente em prever apenas os teores solúveis de $\mathrm{Pb}$, como pode ser observado pelas correlações altamente significativas (Quadro 4). Vários trabalhos têm ressaltado a baixa eficiência dos ácidos orgânicos em solubilizar metais recalcitrantes no solo, especialmente Pb (Renella et al., 2004; Kos \& Lestan, 2004).

\section{CONCLUSÕES}

1. O fosfato natural de Gafsa apresentou os maiores teores de $\mathrm{Cd}$ e $\mathrm{Pb}$ na sua constituição. Entre os fertilizantes fosfatados acidulados, o superfosfato simples mostrou o maior teor de $\mathrm{Cd}$ e $\mathrm{Pb}$. $\mathrm{O}$ termofosfato Yoorin apresentou maior teor de $\mathrm{Pb}$ do que os acidulados.

Quadro 3. Teores de Cd e Pb extraídos por ácido cítrico e DTPA no solo, em dois cultivos sucessivos, em função das doses dos fertilizantes superfosfato simples (SS), superfosfato triplo (ST), termofosfato Yoorin (TY), fosfato de Araxá (FA) e fosfato de Gafsa (FG)

\begin{tabular}{|c|c|c|c|c|c|c|}
\hline \multirow{2}{*}{ Fonte } & \multicolumn{4}{|c|}{ Dose $\left(\mathbf{k g ~ h a} \mathbf{h}^{-1}\right)$} & \multirow{2}{*}{ Equação } & \multirow{2}{*}{$\mathbf{R}^{2}$} \\
\hline & 100 & 300 & 500 & 800 & & \\
\hline \multicolumn{7}{|c|}{ Disponível, mg kg-1 } \\
\hline & \multicolumn{4}{|c|}{ Ácido cítrico } & \multicolumn{2}{|l|}{ Cádmio } \\
\hline SS & $\begin{array}{l}0,03 \\
0,08\end{array}$ & $\begin{array}{l}0,05 \\
0,08\end{array}$ & $\begin{array}{l}0,07 \\
0,24\end{array}$ & $\begin{array}{l}0,10 \\
0,36\end{array}$ & $\begin{array}{l}\mathrm{Cd} 1^{\circ} \text { cultivo }=0,0001^{*} \mathrm{x}+0,0186 \\
\mathrm{Cd} 2^{\circ} \text { cultivo }=0,0004^{*} \mathrm{x}+0,0392\end{array}$ & $\begin{array}{l}0,89 * * \\
0,91 * *\end{array}$ \\
\hline $\mathrm{ST}$ & $\begin{array}{l}0,02 \\
0,07\end{array}$ & $\begin{array}{l}0,04 \\
0,15\end{array}$ & $\begin{array}{l}0,06 \\
0,23\end{array}$ & $\begin{array}{l}0,09 \\
0,35\end{array}$ & $\begin{array}{l}\mathrm{Cd} 1^{\circ} \text { cultivo }=0,0001 x^{* *}+0,0109 \\
\mathrm{Cd} 2^{\circ} \text { cultivo }=0,0004^{* *} \mathrm{x}+0,0323\end{array}$ & $\begin{array}{l}0,94^{* *} \\
0,95^{* *}\end{array}$ \\
\hline $\mathrm{TY}$ & $\begin{array}{l}0,02 \\
0,08\end{array}$ & $\begin{array}{l}0,02 \\
0,16\end{array}$ & $\begin{array}{l}0,04 \\
0,24\end{array}$ & $\begin{array}{l}0,09 \\
0,36\end{array}$ & $\begin{array}{l}\mathrm{Cd} 1^{\circ} \text { cultivo }=0,0001^{* *} \mathrm{x}+0,0134 \\
\mathrm{Cd} 2^{\circ} \text { cultivo }=0,0004^{* *} \mathrm{x}+0,0384\end{array}$ & $\begin{array}{l}0,92^{* *} \\
0,94^{* *}\end{array}$ \\
\hline FA & $\begin{array}{l}0,02 \\
0,10\end{array}$ & $\begin{array}{l}0,05 \\
0,16\end{array}$ & $\begin{array}{l}0,07 \\
0,22\end{array}$ & $\begin{array}{l}0,10 \\
0,31\end{array}$ & $\begin{array}{l}\mathrm{Cd} 1^{\circ} \text { cultivo }=0,0001^{*} \mathrm{x}+0,0022 \\
\mathrm{Cd} 2^{\circ} \text { cultivo }=0,0003^{*} \mathrm{x}+0,0674\end{array}$ & $\begin{array}{l}0,87^{*} \\
0,85^{*}\end{array}$ \\
\hline $\mathrm{FG}$ & $\begin{array}{l}0,09 \\
0,28\end{array}$ & $\begin{array}{l}0,15 \\
0,46\end{array}$ & $\begin{array}{l}0,21 \\
0,64\end{array}$ & $\begin{array}{l}0,30 \\
0,91\end{array}$ & $\begin{array}{l}\mathrm{Cd} 1^{\circ} \text { cultivo }=0,0003^{*} \mathrm{x}+0,0579 \\
\mathrm{Cd} 2^{\circ} \text { cultivo }=0,0009^{*} \mathrm{x}+0,1010\end{array}$ & $\begin{array}{l}0,84^{*} \\
0,83^{*}\end{array}$ \\
\hline SS & $\begin{array}{l}2,28 \\
1,64\end{array}$ & $\begin{array}{l}3,24 \\
2,46\end{array}$ & $\begin{array}{l}4,20 \\
3,28\end{array}$ & $\begin{array}{l}5,64 \\
4,51\end{array}$ & $\begin{array}{l}\text { Chumbo } \\
\mathrm{Pb} 1^{\circ} \text { cultivo }=0,0048^{\circ} \mathrm{x}+1,7997 \\
\mathrm{~Pb} \mathrm{2} 2^{\mathrm{o}} \text { cultivo }=0,0041^{\circ} \mathrm{x}+1,2330\end{array}$ & $\begin{array}{l}0,84^{*} \\
0,82^{*}\end{array}$ \\
\hline $\mathrm{ST}$ & $\begin{array}{l}2,25 \\
1,42\end{array}$ & $\begin{array}{l}3,61 \\
2,54\end{array}$ & $\begin{array}{l}4,97 \\
3,66\end{array}$ & $\begin{array}{l}7,01 \\
5,34\end{array}$ & $\begin{array}{l}\mathrm{Pb} 1^{\circ} \text { cultivo }=0,0068^{*} \mathrm{x}+1,5729 \\
\mathrm{~Pb} 2^{\mathrm{o}} \text { cultivo }=0,0056^{* *} \mathrm{x}+0,8621\end{array}$ & $\begin{array}{l}0,85^{*} \\
0,90^{*}\end{array}$ \\
\hline $\mathrm{TY}$ & $\begin{array}{l}2,16 \\
1,57\end{array}$ & $\begin{array}{l}3,12 \\
2,39\end{array}$ & $\begin{array}{l}4,08 \\
3,21\end{array}$ & $\begin{array}{l}5,52 \\
4,44\end{array}$ & $\begin{array}{l}\mathrm{Pb} 1^{\circ} \text { cultivo }=0,0048^{\circ} \mathrm{x}+1,6768 \\
\mathrm{~Pb} 2^{\circ} \text { cultivo }=0,0041^{*} \mathrm{x}+1,1574\end{array}$ & $\begin{array}{l}0,86^{*} \\
0,89^{*}\end{array}$ \\
\hline FA & $\begin{array}{l}1,41 \\
1,17\end{array}$ & $\begin{array}{l}2,43 \\
1,91\end{array}$ & $\begin{array}{l}3,45 \\
2,65\end{array}$ & $\begin{array}{l}4,98 \\
3,76\end{array}$ & $\begin{array}{l}\mathrm{Pb} 1^{\circ} \text { cultivo }=0,0051^{*} \mathrm{x}+0,9011 \\
\mathrm{~Pb} 2^{\circ} \text { cultivo }=0,0037^{*} \mathrm{x}+0,8045\end{array}$ & $\begin{array}{l}0,85^{*} \\
0,82^{*}\end{array}$ \\
\hline $\mathrm{FG}$ & $\begin{array}{l}1,77 \\
1,17\end{array}$ & $\begin{array}{l}3,09 \\
2,19\end{array}$ & $\begin{array}{l}4,41 \\
3,21\end{array}$ & $\begin{array}{l}6,39 \\
4,74\end{array}$ & $\begin{array}{l}\mathrm{Pb} 1^{\circ} \text { cultivo }=0,0066^{*} \mathrm{x}+1,1143 \\
\mathrm{~Pb} 2^{\circ} \text { cultivo }=0,0049^{*} \mathrm{x}+0,6626\end{array}$ & $\begin{array}{l}0,86^{*} \\
0,92^{* *}\end{array}$ \\
\hline
\end{tabular}

$* *, * \mathrm{e}^{\mathrm{o}}$ : significativos a 1,5 e $10 \%$, respectivamente.

Quadro 4. Correlações de Pearson entre os teores de Cd e Pb na parte aérea de milho e os teores disponíveis no solo, extraídos por ácido cítrico e DTPA

\begin{tabular}{|c|c|c|c|c|c|}
\hline \multirow{2}{*}{ Metal pesado } & \multicolumn{2}{|c|}{ Ácido cítrico } & & \multicolumn{2}{|c|}{ DTPA } \\
\hline & $1^{\circ}$ Cultivo & $2^{\circ}$ Cultivo & & $1^{\circ}$ Cultivo & $2^{\circ}$ Cultivo \\
\hline $\mathrm{Cd}$ & $0,90 * *$ & $0,98^{* *}$ & Superfosfato Simples & nd & nd \\
\hline $\mathrm{Pb}$ & nd & nd & & $0,96^{* *}$ & $0,86^{* *}$ \\
\hline $\mathrm{Cd}$ & $0,85^{* *}$ & $0,88^{* *}$ & Superfosfato Triplo & nd & nd \\
\hline $\mathrm{Pb}$ & nd & nd & & $0,84^{*}$ & $0,79 *$ \\
\hline $\begin{array}{l}\mathrm{Cd} \\
\mathrm{Pb}\end{array}$ & $\begin{array}{c}0,91^{* *} \\
\text { nd }\end{array}$ & $\begin{array}{c}0,94^{*} \\
\text { nd }\end{array}$ & Termofosfato de Yoorin & $\begin{array}{c}\text { nd } \\
0,92^{* *}\end{array}$ & $\begin{array}{c}\text { nd } \\
0,96^{* *}\end{array}$ \\
\hline $\begin{array}{l}\mathrm{Cd} \\
\mathrm{Pb}\end{array}$ & $\begin{array}{c}0,83^{* *} \\
\text { nd }\end{array}$ & $\begin{array}{c}0,84^{* *} \mathrm{~F} \\
\text { nd }\end{array}$ & Fosfato Natural de Araxá & $\begin{array}{c}\text { nd } \\
0,90^{* *}\end{array}$ & $\begin{array}{c}\text { nd } \\
0,91^{* *}\end{array}$ \\
\hline $\begin{array}{l}\mathrm{Cd} \\
\mathrm{Pb}\end{array}$ & $\begin{array}{c}0,87^{* *} \\
\text { nd }\end{array}$ & $\begin{array}{l}0,91^{* *} \mathrm{~F} \\
\text { nd }\end{array}$ & Fosfato Natural de Gafsa & $\begin{array}{c}\text { nd } \\
0,95^{* *}\end{array}$ & $\begin{array}{r}\text { nd } \\
0,83^{*}\end{array}$ \\
\hline
\end{tabular}

**,* $\mathrm{e}^{\mathrm{o}}$ : significativos a 1,5 e $10 \%$, respectivamente; nd: não detectado. 
2. Os maiores teores de $\mathrm{Pb}$ na parte aérea do milho foram fornecidos pelo fosfato de Gafsa no primeiro cultivo. O fosfato de Gafsa também foi responsável pelos maiores teores de $\mathrm{Cd}$ nas plantas no segundo cultivo.

3. O ácido cítrico foi mais eficiente em prever os teores disponíveis de $\mathrm{Cd}$ para a planta de milho, enquanto o DTPA estimou melhor os teores de $\mathrm{Pb}$. Isso mostra a dificuldade na seleção de um único extrator para avaliação da biodisponibilidade de metais.

\section{AGRADECIMENTOS}

O primeiro autor agradece ao CNPq, pela concessão da bolsa de estudos para o curso de doutorado. Os autores agradecem ao Editor Assistente, pelas observações, que melhoraram o trabalho.

\section{LITERATURA CITADA}

AGBENIN, J.O. The distribution and dynamics of chromium and nickel in cultivated and uncultivated semi-arid soils from Nigeria. Sci. Total Environ., 300:189-199, 2002.

AGENCY FOR TOXIC SUBSTANCES AND DISEASE REGISTRY - ATSDR. Priority list of hazardous substances. Disponível em: <http://www.atsdr.cdc.gov/cercla/ 05list.html>. Acesso em 18 maio de 2009.

AMARAL SOBRINHO, N.M.B.; COSTA, L.M.; OLIVEIRA, C. \& VELlOSO, A.C.X. Metais pesados em alguns fertilizantes e corretivos. R. Bras. Ci. Solo, 16:271-276, 1992.

ASSOCIATION OF FERTILIZERS AND PHOSPHATE CHEMISTS - AFPC. Disponível em: < http://www.afpc.net/ Metal\%20Regulations.html>. Acesso em 19 nov. de 2006.

BEDIN, I.; FURTINI NETO, A.E.; RESENDE, A.V.; FAQUIN, V.; TOKURA, A.M. \& SANTOS, J.Z.L. Fertilizantes fosfatados e produção da soja em solos com diferentes capacidades tampão de fosfato. R. Bras. Ci. Solo, 27:639646, 2003.

BIZARRO, V.G.; MEURER, E.J. \& TATSCH, F.R.P. Teor de cádmio em fertilizantes fosfatados comercializados no Brasil. Ci. Rural, 38:247-250, 2008.

BRAGA, N.R.; MASCARENHAS, H.A.A.; BULISANI, E.A.; RAIJ, B.van; FEITOSA, C.T. \& HIROCE, R. Eficiência agronômica de nove fosfatos em quatro cultivos consecutivos de soja. R. Bras. Ci. Solo, 15:315-319, 1991.

BRASIL. Ministério da Agricultura, Pecuária e Abastecimento. Sistema de legislação agrícola federal. Disponível em: $<$ http://extranet.agricultura.gov.br/sislegis-consulta/ servlet/VisualizarAnexo?id=11365>. Acesso em: 19 jun. de 2008.
CAMARGO, M.S.; ANJOS, A.R.M.; ROSSI, C. \& MALAVOLTA, E. Adubação fosfatada e metais pesados em Latossolo cultivado com arroz. Sci. Agric., 57:513-518, 2000.

CHIEN, S.H.; CARMONA, G.; PROCHNOW, L.I. \& AUSTIN, E.R. Cadmium availability from granulated and bulkblended phosphate-potassium fertilizers. J. Environ. Qual., 32:1911-1914, 2003.

COMPANHIA DE TECNOLOGIA DE SANEAMENTO AMBIENTAL - CETESB. Valores orientadores para solos e águas subterrâneas no Estado de São Paulo. Disponível em: <http://www.agsolve.com.br/pdf/artigos/ novatabelacetesb2005.pdf $>$. Acesso em: 18 maio de 2009.

COTTER-HOWELLS, J. \& CAPORN, S. Remediation of contaminated land by formation of heavy metal phosphates. Appl. Geochem., 11:335-342, 1996.

EMPRESA BRASILEIRA DE PESQUISA AGROPECUÁRIA EMBRAPA. Manual de métodos de análises de solo. Brasília, Embrapa Comunicação para Transferência de Tecnologia, 1997. 212p.

EMPRESA BRASILEIRA DE PESQUISA AGROPECUÁRIA EMBRAPA. Manual de análises químicas de solos, plantas e fertilizantes. Brasília, Embrapa Comunicação para Transferência de Tecnologia, 1999. 370p.

GONÇALVES JÚNIOR, A.C.; LUCHESE, E.B. \& LENZI, E. Avaliação da fitodisponibilidade de cádmio, chumbo e crômio, em soja cultivada em Latossolo Vermelho Escuro tratado com fertilizantes comerciais. Química Nova, 23:173-177, 2000.

KAMINSKI J. \& PERUZZO, G. Eficácia de fosfatos naturais reativos em sistemas de cultivo. Santa Maria, NRS-SBCS, 1997. 31p. (Boletim Técnico, 3)

KOS, B. \& LESTAN, D. Chelator induce phytoextraction and in situ soil washing of $\mathrm{Cu}$. Environ. Pollut., 132:333-339, 2004.

KRISHNAMURTI, G.S.; HUANG, P.M. \& KOZAK, L.M. Sorption and desorption kinetics of cadmium from soils: Influence of phosphate. Soil Sci., 164:888-898, 1999.

LANGENBACH, T. \& SARPA, M. Teor de cádmio nos fertilizantes fosfatados brasileiros. R. Bras. Ci. Solo, 9:179181,1985 .

LINDSAY, W.L. \& NORVELL, W.A. Development of a DTPA soil test for zinc, iron, manganese and copper. Soil Sci. Soc. Am. J., 42:421-428, 1978.

McBRIDE, M.B. \& SPIERS, G. Trace element content of selected fertilizers and dairy manures as determined by ICP-MS. Comm. Soil Sci. Plant Anal., 32:139-156, 2001.

McLAUGHLIN, M.J. \& SINGH, B.R. Cadmium in soil and plants. Dordrecht, Kluwer Academic, 1999. 364p.

MENDES, A.M.S.; DUDA, G.P.; NASCIMENTO, C.W.A. \& SILVA, M.O. Bioavailability of cadmium and lead in a soil amended with phosphorus fertilizers. Sci. Agric., 63:328332, 2006.

MORTVEDT, J.J. Cadmium levels in soils and plants from some long term soil fertility experiments in the United States of America. J. Environ. Quality, 16:137-142, 1987. 
NASCIMENTO, C.W.A.; FONTES, R.L.F. \& NEVES, J.C.L. Dessorção, extração e fracionamento de manganês em Latossolos. R. Bras. Ci. Solo, 26:589-597, 2002.

NRIAGU, J.O. \& PACYNA, J.M. Quantitative assessment of worldwide contamination of air, water and soils with trace metals. Nature, 333:134-139, 1988.

OTERO, N.; VITÒRIA, L.; SOLER, A. \& CANALS, A. Fertilizer characterization: Major, trace and rare earth elements. Applied Geochem., 20:1473-1488, 2005.

PROCHNOW, L.I. Fertilizantes fosfatados: Algumas crenças e alguns fatos científicos. Disponível em: <http:// www.manah.com.br/informativos.asp?idI=12>. Acesso em 18 maio de 2009.
PROCHNOW, L.I.; PLESE, L.M. \& ABREU, M.F. Bioavailability of cadmium contained in single superphosphates produced from different Brazilian raw materials. Comm. Soil Sci. Plant Anal., 32:283-294, 2001.

RENELLA, G.; LANDI, L.L. \& NANNIPIERI, P. Degradation of low molecular weight organic acids complexed with heavy metals in soil. Geoderma, 122:311-315, 2004.

WESTFALL, D.G.; MORTVEDT, J.J.; PETERSON, G.A. \& GANGLOFF, W.J. Efficient and environmentally safe use of micronutrients in agriculture. Comm. Soil Sci. Plant Anal., 36:169-182, 2005. 